mit der Temperatur analog zum Terephthalsäuredimethylester stark zu. Deshalb ist eine Reinigung der Substanz von $\mathrm{C}_{6} \mathrm{H}_{4}\left(\mathrm{CO}_{2} \mathrm{CH}_{3}\right)_{2}$ umständlich und zeitraubend. Aus diesem Grund erfolgt die Umsetzung am zweckmäßigsten mit einem Überschuß an $\mathrm{Cr}(\mathrm{CO})_{6}$, damit das $\mathrm{C}_{6} \mathrm{H}_{4}\left(\mathrm{CO}_{2} \mathrm{CH}_{3}\right)_{2}$ vollkommen durchreagieren kann.

Terepht ha ls äu redigly k o lest e r chrom tric a r bo n y l: $500 \mathrm{mg} \mathrm{Cr}(\mathrm{CO})_{6}(2 \mathrm{mMole})$ und $600 \mathrm{mg}$ Terephthalsäurediglykolester (2,3 mMole) gelöst in $40 \mathrm{ml} \mathrm{Di-} n$-butyläther wurden in der oben erwähnten Apparatur bei einer Badtemperatur von $190^{\circ}$ in einer $\mathrm{N}_{2}$-Atmosphäre zur Reaktion gebracht. Nach $6 \mathrm{Stdn}$. hatten sich $150 \mathrm{ml}$ Kohlenmonoxyd abgespalten. Die Reaktion wurde abgebrochen, da längere Reaktionszeiten die Ausbeuten verringern. Die orange-rote Reaktionslösung wurde auf Raumtemperatur gebracht, filtriert und das Lösungsmittel am Rotationsverdampfer abgezogen. Das feste, orange Rohprodukt wurde in $30 \mathrm{ml}$ siedendem Wasser gelöst, die Lösung heiß fil-

\section{Cyclopentadienyltrimethylplatinum (IV)}

\author{
By S. D. Robinson and B. L. Shaw
}

School of Chemistry, The University, Leeds, 2

(Z. Naturforschg. 18 b, 507 [1963] ; eingegangen am 18. März 1963)

Platinum is unique among the metals in the number and stability of complexes it forms with hydrocarbon ligands, viz., olefins, acetylenes, alkyl and aryl groups, etc. However, only one cyclopentadienyl complex is known - allylcyclopentadienylplatinum (II) ${ }^{1}$-in spite of several attempts to make such complexes. We now find that trimethylplatinum iodide ${ }^{2}$ with cyclopentadienylsodium in tetrahydrofuran gives cyclopentadienyltrimethylplatinum (IV) as air stable white prisms, m.p. $65^{\circ}$. It is very soluble in common organic solvents and may be recrystallised from methyl alcohol, but is best purified by sublimation at $25^{\circ} / 10^{-2} \mathrm{~mm}$. Cyclopentadinyltrimethylplatinum (IV) is so volatile that a small

1 B. L. Shaw and N. Sheppard, Chem. and Ind. 1961, 517.

2 W. J. Pope and S. J. Peachey, J. chem. Soc. [London] 95, 571 [1909]. triert und das Filtrat im Kühlschrank abkühlen gelassen. Nach 5 Stdn. schieden sich rote Nadeln und noch etwas weißes Ausgangsprodukt ab. Beide Substanzen wurden dann mit $30 \mathrm{ml}$ Wasser von $45^{\circ}$ durchgerührt, wobei Terephthalsäurediglykolester in Lösung ging, während die rote Substanz zurück blieb. Sie wurde nochmals aus $10 \mathrm{ml}$ heißem Wasser umkristallisiert. Ausbeute: $35 \mathrm{mg}$ [ $4 \%$ bezogen auf $\left.\mathrm{Cr}(\mathrm{CO})_{6}\right]$.

$\left(\mathrm{HOCH}_{2} \mathrm{CH}_{2} \mathrm{CO}_{2}\right)_{2} \mathrm{C}_{6} \mathrm{H}_{4} \mathrm{Cr}(\mathrm{CO})_{3}$ (390).

$$
\text { Ber. C } 46,15 \text { H } 3,58 \text { Cr } 13,3 \text {, }
$$$$
\text { Gef. C } 46,38 \text { H } 3,75 \text { Cr } 13,0 \text {. }
$$

Eigenschaften : rote Kristalle, welche bei $137^{\circ}$ (K of le r-Bank) schmelzen. Die Verbindung ist an der Luft beständig. Sie kann nicht sublimiert werden und ist gut löslich in Methanol, Äthanol, Aceton, Äther und heißem Wasser, mäßig löslich in Benzol und unlöslich in Heptan.

Dem Fonds der Chemischen Industrie und der Deut. $\mathrm{schen}$ Forschungsgemeinschaft sei für die finanzielle Unterstützung dieser Arbeit gedankt.

sample placed in an open laboratory volatilised completely in about 20 min. [Found: C, 31.65; H, 4.65; M., cryoscopically in $0.97 \% \mathrm{w} / \mathrm{w}$ benzene solution, 304 , in $1.47 \%$ solution, $294 . \mathrm{C}_{8} \mathrm{H}_{14} \mathrm{Pt}$ requires $\mathrm{C}, 31.45$; H, $4.6 \%$; M. 305.]

The molecular weight data show cyclopentadienyltrimethylplatinum (IV) to be a monomer, in contrast to tetramethylplatinum (IV) which is a tetramer ${ }^{3}$. We think that the cyclopentadienyl group is not $\sigma$-bonded to the metal but is 'sandwich'-bonded, thus giving the platinum atom an inert gas electron configuration.

The N.M.R. spectrum (in carbon tetrachloride) is particularly valuable in confirming the structure, showing two main peaks, with relative intensities of $9: 5$, at $\tau=9.12$ (methyl protons) and $\tau=4.39$ (cyclopentadienyl protons). Each of these main peaks has two symmetrically placed side peaks due to coupling with the magnetic ${ }^{195} \mathrm{Pt}$ nucleus (natural abundance $33 \%$ ) with coupling constants $82.5 \mathrm{c}$./sec. (methyl protons) and $5.8 \mathrm{c}$./sec. (cyclopentadienyl protons).

${ }^{3}$ R. E. Rundle and J. H. Sturdivant, J. Amer. chem. Soc. 69, 156 [1947].
Beiträge zur Chemie des Schwefels, $66^{1}$

Über die Darstellung und Eigenschaften der Diäthylester der Penta- und Hexasulfandicarbonsäuren sowie der entsprechenden Monothiocarbonsäuren

Von F. Fehér, W. Becher, R. Kreutz und F.-R. Minz

Institut für Anorganische Chemie der Universität Köln (Z. Naturforschg. 18 b, 507-508 [1963] ; eingegangen am 9. Februar 1963)

Im Verlauf unserer Arbeiten mit den höheren Chlorsulfanen brachten wir $\mathrm{S}_{3} \mathrm{Cl}_{2}$ und $\mathrm{S}_{4} \mathrm{Cl}_{2}$ mit den thio- kohlensauren Salzen $\mathrm{C}_{2} \mathrm{H}_{5} \mathrm{O} \cdot \mathrm{CO} \cdot \mathrm{SK}$ und $\mathrm{C}_{2} \mathrm{H}_{5} \mathrm{O} \cdot \mathrm{CS} \cdot \mathrm{SK}$ zur Umsetzung. Im wesentlichen hielten wir uns dabei an eine Arbeitsvorschrift von Twiss ${ }^{2}$, der die niederen Homologen $\mathrm{S}_{\mathrm{x}}\left(\mathrm{CO} \cdot \mathrm{OC}_{2} \mathrm{H}_{5}\right)_{2}$ und $\mathrm{S}_{\mathrm{x}}\left(\mathrm{CS} \cdot \mathrm{OC}_{2} \mathrm{H}_{5}\right)_{2}(x=$ $3 ; 4)$ unter Verwendung von $\mathrm{SCl}_{2}$ und $\mathrm{S}_{2} \mathrm{Cl}_{2}$ darstellte.

Alle Reaktionen führten wir nach folgendem Schema durch: 0,1 Mol thiokohlensaures Salz wurde in $100 \mathrm{ml}$ über Natrium getrocknetem Äther unter lebhaftem Rühren suspendiert. Dazu wurde unter Kühlung des Reak-

1 65. Mitt.: F. F 849 [1962].

2 D. Twiss, J. Amer. chem. Soc. 49, 491 [1927]. 
tionskolbens mit Eiswasser aus einem Tropftrichter $0,05 \mathrm{Mol}$ Chlorsulfan (in $50 \mathrm{ml}$ trockenem Äther gelöst) langsam zugetropft. Nach Beendigung des Eintropfens wurde das Kühlbad entfernt und die Reaktionsmischung noch $1-2$ Stdn. bei Zimmertemperatur nachreagieren lassen. Anschließend wurde das gebildete $\mathrm{KCl}$ auf einer Glasnutsche von der ätherischen Lösung getrennt; der Äther wurde dann im Wasserstrahlvakuum, später im Hochvakuum entfernt.

Wegen der Empfindlichkeit der Chlorsulfane gegen Alkali wurden die Reaktionsgefäße vorher mit konz.Salzsäure ausgekocht. Die Umsetzungen verliefen quantitativ. Es wurde darauf geachtet, daß das Chlorsulfan nicht im Überschuß zugegeben wurde (geringste Spuren sind leicht an dem charakteristischen scharfen Geruch zu erkennen), da dies die Haltbarkeit der Reaktionsprodukte herabsetzt. Die so hergestellten Substanzen sind schwach gelblich-grüne bis gelbe Öle von angenehmem Geruch, die keine Neigung zur Kristallisation zeigen. Nach mehrwöchigem Aufbewahren bei ca. $-5{ }^{\circ} \mathrm{C}$ waren noch keine Anzeichen von Zersetzung ${ }^{3}$ zu erkennen. Entsprechend den von Twiss ${ }^{2}$ dargestellten niede-

${ }^{3}$ D. Twiss berichtet, daß seine Substanzen vom Typus $\mathrm{S}_{\mathrm{X}}\left(\mathrm{CO} \cdot \mathrm{OC}_{2} \mathrm{H}_{5}\right)_{2}$ langsam unter Abscheidung von Schwefel in die Disulfide übergingen.

\section{Trimethylgermanyl-perchlorat}

Von Ingeborg Ruidisch und Max Schmidt

Institut für Anorganische Chemie der Universität Marburg (Z. Naturforschg. 18 b, 508-509 [1963] ; eingegangen am 3. April 1963)

Zur Darstellung verschiedener Ester des in freier Form nicht isolierbaren Trimethylgermanols ${ }^{1}$ mit anorganischen Sauerstoffsäuren haben wir Trimethylchlorgerman ${ }^{1}$ mit wasserfreien Säuren umgeestert ${ }^{2}$, Hexamethyldigermoxan mit Säureanhydriden gespalten ${ }^{3}$ oder Trimethylchlorgerman mit Silbersalzen umgesetzt ${ }^{3}$. Für die Synthese des Perchlorsäureesters ${ }^{4}$ eignet sich nur die letztgenannte Methode, da der nach dem ersten Verfahren nach

$$
\left(\mathrm{CH}_{3}\right)_{3} \mathrm{GeCl}+\mathrm{HClO}_{4} \rightarrow\left(\mathrm{CH}_{3}\right)_{3} \mathrm{GeOClO}_{3}+\mathrm{HCl}
$$

entwickelte Chlorwasserstoff die freie Perchlorsäure reduziert und das für den zweiten Reaktionsweg

$\left(\mathrm{CH}_{3}\right)_{3} \mathrm{GeOGe}\left(\mathrm{CH}_{3}\right)_{3}+\mathrm{Cl}_{2} \mathrm{O}_{7} \rightarrow 2\left(\mathrm{CH}_{3}\right)_{3} \mathrm{GeOClO}_{3}$

erforderliche Dichlorheptoxid explosionsartig mit dem Ausgangsmaterial reagiert.

Silberperchlorat reagiert dagegen rasch und quantitativ bei Zimmertemperatur mit Trimethylchlorgerman in

1 M. Schmidt u. I. Ruidisch, Z. anorg. allg. Chem. 311, 331 [1961].

2 M. Sснмidt u. I. Ruidisch, unveröffentlicht.

3 M. Sсhмidt u. I. Ruidisch, Angew. Chem. 73, 408 [1961];

M. Schmidt, H. Schmidbaur u. I. Ruidisch, Angew. Chem.

73, 408 [1961]; M. Schmidt, H. Schmidbaur, I. Ruidisch u. ren Homologen sind auch unsere Substanzen nicht destillierbar. Zur Charakterisierung wurden UV- und IR-Spektren aufgenommen sowie die Brechungsindizes und Dichten bestimmt.

Nach dem beschriebenen Verfahren wurden die folgenden Sulfanderivate dargestellt:

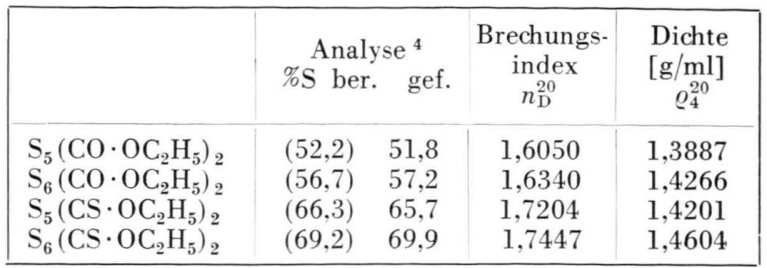

Bei allen Ausgangssubstanzen wurde Wert auf hohe Reinheit ${ }^{5}$ gelegt, um von dieser Seite her eine Verunreinigung der gewonnenen Produkte auszuschließen. In den Endprodukten ließen sich ir-spektroskopisch keine Spuren der eingesetzten Substanzen mehr nachweisen.

${ }^{4}$ Die Schwefelbestimmung wurde nach der Methode von W u r z s c h mit t durchgeführt.

5 Bezüglich der Chlorsulfane vgl. G. Brauer, Handbuch d. präp. anorg. Chem., Bd. I, 2. Aufl., S. 338, 340. benzolischer Lösung nach

$$
\left(\mathrm{CH}_{3}\right)_{3} \mathrm{GeCl}+\mathrm{AgClO}_{4} \rightarrow\left(\mathrm{CH}_{3}\right)_{3} \mathrm{GeOClO}_{3}+\mathrm{AgCl} \text {. }
$$

Nach Abtrennen vom ausgefallenen Silberchlorid und Abziehen des Benzols im Vakuum bei $20^{\circ}$ bleibt der rohe Ester als rotbraune Flüssigkeit zurück. Vorsichtige Vakuumdestillation bei $91-92^{\circ} / 2 \mathrm{~mm}$ liefert die Verbindung rein.

Reines Trimethylgermanyl-perchlorat stellt eine farblose, klare, leicht bewegliche und stechend riechende Flüssigkeit dar, die an feuchter Luft stark raucht. Kleine Mengen $(1-2 \mathrm{~g})$ können bei $91-92{ }^{\circ} \mathrm{C} / 2 \mathrm{~mm}$ unzersetzt destilliert werden. Die Destillation muß jedoch immer unter größter Vorsicht erfolgen, da schon bei geringfügiger Zersetzung nach

$$
2\left(\mathrm{CH}_{3}\right)_{3} \mathrm{GeOClO}_{3} \rightarrow\left[\left(\mathrm{CH}_{3}\right)_{3} \mathrm{Ge}\right]_{2} \mathrm{O}+\mathrm{Cl}_{2} \mathrm{O}_{7}
$$

Dichlorheptoxid mit den Methylgruppen explosionsartig reagieren kann. Unter Feuchtigkeitsausschluß aufbewahrtes farbloses Trimethylgermanyl-perchlorat verfärbt sich nach einigen Tagen zunächst gelb, dann braun und im Verlauf mehrerer Wochen zuletzt schwarz, wobei es gleichzeitig zur Abscheidung fester Oxydationsprodukte kommt. Das Öffnen solcher gealterter

P. Bormmann, Angew. Chem. 73, 408 [1961] ; M. Schmidt, I. Ruidisch u. H. Schmidbaur, Chem. Ber. 94, 2451 [1961]; M. Sснмidt u. I. Ruidisch, Chem. Ber. 95, 1434 [1962].

4 Die analoge Siliziumverbindung wurde von U. Wannagat, F. Brandmair, W. Liehr u. H. Niederprüm dargestellt, Z. anorg. allg. Chem. 302, 185 [1959]. 